\title{
Adrenal myelolipoma: a comprehensive review
}

Ábel Decmann ${ }^{1}$, Pál Perge $^{1}$, Miklós Tóth ${ }^{1}$, Peter Igaz $^{1,2 *}$

${ }^{1} 2^{\text {nd }}$ Department of Medicine, Semmelweis University, 1088 Budapest, Szentkirályi str. 46. Hungary

${ }^{2}$ Molecular Medicine Research Group, Hungarian Academy of Sciences and Semmelweis University, 1088 Budapest, Szentkirályi str. 46., Hungary

*Corresponding author: Dr.Peter Igaz MD MSc PhD DSc

$2^{\text {nd }}$ Department of Medicine, Faculty of Medicine, Semmelweis University, H-1088 Budapest, Szentkirályi str. 46., Hungary

Phone/Fax +36-1-2660816

E-mail: igaz.peter@med.semmelweis-univ.hu

\section{Available ORCID IDs of authors:}

Ábel Decmann: orcid.org/0000-0002-4635-8644

Pál Perge: orcid.org/0000-0002-0722-7684

Peter Igaz: orcid.org/0000-0003-2192-554X

Total word count excluding title page abstract, references and figure legends: 3314 


\begin{abstract}
:
Adrenal myelolipoma is an invariably benign neoplasm of the adrenal gland that is the second most common primary adrenal incidentaloma following adrenocortical adenomas. It is composed of elements of adipose tissue and extramedullary hematopoiesis. Hypotheses on stem cells and hormonal factors have been formulated regarding its pathogenesis that is still obscure. Despite its benign behaviour, adrenal myelolipoma is clinically relevant as it might cause significant difficulties in the differential diagnosis of adrenal tumors. We have reviewed 420 cases reported between 1957 and 2017 on adrenal myelolipoma retrieved from PubMed and Scopus databases and also 20 of our case series to provide a comprehensive analysis of their pathology, epidemiological and clinical features. The average age for its diagnosis was 51 years, and no gender difference was observed. The average size of tumors was $10.2 \mathrm{~cm}$. Congenital adrenal hyperplasia was associated to $10 \%$ of all cases analyzed, while other adrenal hypersecretory disorders (cortisol, aldosterone) were found in $7.5 \%$ of cases. Computed tomography and magnetic resonance imaging can be reliably used for its differential diagnosis. If the diagnosis of an adrenal myelolipoma is unambiguous, and no associated symptoms or hormonal activity are established, surgical intervention is usually not necessary.
\end{abstract}

Keywords: adrenal myelolipoma, congenital adrenal hyperplasia, differential diagnosis, management

Abbreviations: ACTH: adrenocorticotropic hormone; AML: adrenal myelolipoma; $\mathrm{CAH}$ : congenital adrenal hyperplasia; EMH: extramedullary hematopoiesis 


\section{Introduction}

Myelolipoma is an invariably benign neoplasm of the adrenal cortex. The tumor is composed of fat and myeloid tissue and is listed in the group of mesenchymal and stromal tumors of the adrenal cortex according to the latest update of World Health Organization on endocrine tumors [1]. Despite its benign behavior, adrenal myelolipoma (AML) is clinically relevant as it might cause significant difficulties in the diagnosis of adrenal neoplasms. The number of case reports on adrenal myelolipomas has greatly increased in the last decades. This phenomenon is probably related to the more advanced and wide-spread use of abdominal imaging modalities [computed tomography $(\mathrm{CT})$ and magnetic resonance imaging (MRI)] leading to the increased incidence of adrenal incidentalomas including AML.

Adrenal myelolipomas are found in one out of 500-1250 autopsy cases [2]. However, it is almost impossible to assess the exact clinical prevalence of the tumor because of the high percentage of asymptomatic cases and its benign behavior. Following the adrenocortical adenoma accounting for $60-70 \%$ of adrenal incidentalomas, AML is the second most common primary adrenal incidentaloma representing $6-16 \%$ of adrenal incidentalomas [3-5]. Myelolipomas can rarely appear at other sites of the human body, as well, hence the term extra-adrenal myelolipoma. Among numerous locations, myelolipomas have been described in the mediastinum [6], spleen [7], kidney [8], bones [9], thorax [10], nasal cavity [11], ectopic adrenal cortex $[12,13]$ as well as at extradural sites [14] or in the eyes [15]. Biochemically, myelolipomas are mostly non-functional.

Our aim has been to review all available case reports and other articles on adrenal myelolipoma in order to present a comprehensive view on this tumor. We have studied 420 histologically verified clinical cases of adrenal myelolipoma found in reviews and case reports of PubMed and Scopus databases from 1957 to 2017. Moreover, we have included 20 
of our documented, histologically verified cases. (Data of all adrenal myelolipoma cases included in our analysis are presented in Supplementary Table 1.) We listed only the cases where detailed clinical information was available and that could be clearly differentiated from each other and are not only parts of larger series.

\section{Pathogenesis}

Myelolipomas are composed of mature adipocytes and trilinear hematopoietic cells just as the properly functioning bone marrow and the extramedullary hematopoiesis (EMH) caused by chronic hematopoietic disorders. Extramedullary hematopoiesis is a physiologic condition during fetal development until the bone marrow maturates. These spots of hematopoiesis can take place in different organs like liver or spleen. After birth, EMH is mostly associated with pathological processes such as myeloproliferative disorders (e.g. myelofibrosis) or haemoglobinopathies (e.g. thalassemias) [16]. According to our current view, EMH and myelolipoma are two different entities but with nearly the same pathological appearance. Both differ from the bone marrow by the lack of reticular sinusoids. In the general clinical practice, the decision between EMH and myelolipoma is based on clinical features: EMH usually occurs in hematological conditions, while myelolipoma is a tumor that is accompanied by a healthy bone marrow [17]. Myelolipomas are encapsulated or well-demarcated lesions, and are composed of fat cells and have normal marrow hematopoietic elements. On the other hand, extramedullary hematopoiesis is not circumscribed, and fat is not its obligatory component [18].

The pathogenesis of myelolipoma is obscure. It is hypothesized that the primary event is a metaplastic change occuring in the reticuloendothelial cells of the blood capillaries due to stimuli like necrosis, infection or stress [19-21]. One report described the presence of a 
balanced translocation of $(3 ; 21)(\mathrm{q} 25 ; \mathrm{p} 11)$ in the tumor, but its pathogenic relevance is unclear [22]. Bishop et al. found that the majority of myelolipomas had non-random X-chromosome inactivation suggesting the clonal origin of these tumors [23]. Another hypothesis claims that adrenal myelolipoma originates from the interaction of two different kinds of progenitor cells. First, adipose tissue develops from mesenchymal stem cells harbored in the vessel wall of stromal fat of adrenal cortex under certain stimuli. When adipocytes are differentiating and maturing, they become inflammatory and stimulate the adrenal cortex to release factors or substances that recruit circulating hematopoietic progenitors to settle and differentiate [24].

Another major group of hypotheses relates to hormonal pathways. Many decades ago, in experiments by Hans Selye, combined treatment of hypophysectomized rats with methyltestosterone and lyophilized anterior pituitary extracts lead to the transformation of the entire zona fasciculata and zona reticularis into a tissue having very similar characteristics to bone marrow [25]. These findings might correlate with the clinical observation, that some patients with untreated congenital adrenal hyperplasia having very high serum adrenocorticotropin (ACTH) levels develop myelolipoma. A case of ectopic ACTH overproduction due to a lung carcinoma associated with myelolipoma has been reported [26]. However, a Japanese group could not confirm the over-expression of ACTH receptors in the myelolipoma of a CAH patient that argues against ACTH to be a major causative factor [27]. Another hormonal overstimulation theory suggests stimulation via higher level of erythropoietin caused by chronic anemia $[20,28]$.

It might be possible that both altered mesenchymal stem cell functioning and hormonal stimuli act together in the pathogenesis of myelolipoma. 


\section{Pathology}

Common macroscopic findings of adrenal myelolipomas include a capsule or pseudocapsule, yellow and red/brown cut surfaces, and hemorrhage. Capsules and the absence of a capsule were reported in $26 \%$ and $1 \%$ of all reported cases, respectively. However, $73 \%$ of the case reports did not provide any information about capsules in our statistics. Pseudocapsule is an outer layer that contains the compressed zona glomerulosa and zona fasciculata. On the cut surface, yellow color corresponds to the adipose tissue while red or brown color pertains to the hematopoietic tissue. Myelolipomas are round or elliptical tumors with varying diameter from a few millimeters up to 43 centimeters, and with an average of 10.2 centimeters according to our analysis. Tumors exceeding $10 \mathrm{~cm}$ in diameter are called giant myelolipomas [20]. In our analysis, 161 reported tumors belong to this category, which is $35.7 \%$ of all cases. However, giant myelolipomas are more likely to be reported in case reports forming the basis of our analysis, thus their might be a publication bias. Difference in diameter between rightand left-sided tumors was not observed (average right: 10.2 centimeters, average left: 10.3 centimeters). The heaviest reported AML so far was 11400 grams [29]. The weight of the tumors poorly correlates - especially in the bigger ones - with the diameter because of intratumoral hemorrhages and the varying ratio between adipose tissue and hematopoietic tissue (Figure 1.). Hemorrhages on the cut surface were seen and mentioned in $19 \%$ of the cases.

The characteristic microscopic appearance of myelolipoma is usually a dense adipose tissue, which can occasionally vary. The other constant component is trilineage hematopoietic tissue with the abundance of erythroid and granulocytic/lymphoid elements as well as megakaryocytes. Rarely, myelolipomatous foci were found in adrenocortical adenomas or ganglioneuroma [30, 31]. Both associated zona fasciculata and zona glomerulosa hyperplasia was observed in $1 \%$ of all cases each. Zona reticularis hyperplasia was seen in altogether 2 
cases $(0.5 \%)$. In $5.7 \%$ of all cases, there is concomitant adrenocortical adenoma, as well. Association with adrenocortical carcinoma was rarely observed $(0.5 \%)$. Other findings at histopathological examination in association with myelolipoma were osseous metaplasia [32], adrenal nodular hyperplasia, calcification, black pigmented adenoma [33], and hibernoma [34].

\section{Clinical findings}

Based on our analysis, AML is diagnosed at the age of 51 (SD: 14.4) years on average (Figure 2.). There was no remarkable difference between male and female patients, with an average of 50.84 years and 51.02 years, respectively. The youngest patient was 1 year old at the diagnosis [35], while the oldest was 83 years old [36]. Former and recent reviews suggest that the diagnosis of myelolipoma is more likely made in people in their fifties or sixties [37, 38]. 434 out of 440 reports included the gender of the patient. Among these $211(48.6 \%)$ were female and 223 (51.4\%) male, thus no gender difference can be established. In contrast, Lam reported a female preponderance based on two Chinese studies [39].

In 362 cases of 440 analyzed AMLs, the history of the diagnosis was well documented In Table 1., we present the leading complaints of the patients. Except for giant myelolipomas leading to abdominal discomfort by mass effects, it is difficult to unambiguously associate these symptoms with adrenal myelolipoma.

The laterality of tumors corresponded to previous findings describing that right-sided myelolipomas are more frequent $[1,20,39]$. In our analysis, 260 tumors $(59.2 \%)$ were on the right side, 111 on the left side $(25.3 \%)$, while 54 tumors $(12.3 \%)$ were bilateral (no data were available in 15 cases). The left-sided tumors of the bilateral myelolipomas are usually slightly 
larger (average diameter $10.9 \mathrm{~cm}$ on the left, and $9.3 \mathrm{~cm}$ on the right side), however, the difference was not significant $(\mathrm{p}=0.25)$.

Myelolipomas are relatively slowly growing tumors. Observations of Han et al. showed the growths of 13 myelolipomas of 12 patients. Five tumors did not change in size, two tumors shrank and six grew larger. The fastest rate of growth was 5.5\%/month $(4.5 \mathrm{~cm}$ to $5.5 \mathrm{~cm}$ over 4 months), while the average growing speed was $0.64 \%$ per a month [40]. In a most recent study, Campbell et al. found tumor growth in 11 of 69 AML cases, where followup imaging by computed tomography was done at a median time interval of 3.9 years. The median growth per year was $0.16 \mathrm{~cm} /$ year (min: $0.08 \mathrm{~cm} /$ year, max: $0.71 \mathrm{~cm} /$ year), but the authors claimed that the growth pattern of these tumors might not be always constant or predictable [41]. Follow-up of larger AML cohorts would be needed to assess their growth rate that could form the basis for clinical recommendations.

Spontaneous rupture of an AML may result in chronic retroperitoneal hemorrhage or very rarely even in hemorrhagic shock [42]. Rupture of tumor was found in $4.5 \%$ of all cases (20 cases). The median diameter of ruptured tumors was $120 \mathrm{~mm}$. Most of the bleeding adrenal myelolipomas were larger than $10 \mathrm{~cm}$. The smallest AML reported to bleed was 6.5 cm large [36].

Associated disease was found in the majority of patients. 98 patients had hypertension while 41 had type 2 diabetes mellitus.

Endocrine disorders were the most prevalent in myelolipoma patients. Among the 440 patients analyzed, 44 patients had congenital adrenal hyperplasia $(\mathrm{CAH})$ accounting for $10 \%$ of all cases. 35 patients had 21-hydroxylase deficiency (6 salt-wasting, 13 simple virilizing, 3 non-classical, and 19 no further detailed). The second most common form was the 17-alphahydroxylase deficiency with 5 cases. 11-beta-hydroxylase deficiency was only present in one 
case. Among the CAH patients, bilateral myelolipomas were found in almost half of the cases (18 cases, 51\%), 12 myelolipomas were left-sided (34\%), while right-sided tumors were only in minority (5 cases, 14\%), thus this trend is opposite to the non-CAH-associated AML, where right-sided is more frequent. $\mathrm{CAH}$ were newly diagnosed or poorly managed in 26 cases out of 44 .

23 patients had hypercortisolism and nine had primary aldosteronism associated to myelolipoma, In 4 cases, androgen secretion was documented. Hypogonadism, primary hyperparathyroidism and pheochromocytoma were found in two cases each. Other reported endocrine diseases included Carney's complex [43], polycystic ovary syndrome [44], papillary thyroid cancer [45] and hyper- or hypothyroidism, Multiple Endocrine Neoplasia syndrome type 1 [46], and Nelson syndrome [47], but these are unlikely to represent real associations. Complaints and symptoms of hypercortisolism, hyperaldosteronism and hyperandrogenism typically vanished after the myelolipoma was removed. Altogether 33 tumors were observed that produced any kind of adrenal gland hormone apart from cases of $\mathrm{CAH}$, which is $7.5 \%$ of all cases. Moreover, the average size of functioning tumors is smaller $(68.5 \mathrm{~mm})$ than of the non-functional lesions $(104.4 \mathrm{~mm})$ that is possibly related to the earlier diagnosis of hormone-secreting tumors. The observation of adrenal hypersecretory disorders associated with adrenal myelolipoma might indicate that AML is not always a tumor composed purely of elements of adipose and extramedullary hemopoetic tissues, but might even include adrenocortical parts accounting for steroid hypersecretion. Certainly, our analysis of case reports might result in a skewed estimate due to publication bias, and the actual hormonal activity of AML might be somewhat lower.

Non-endocrine disorders were found in 56 patients. Among these $12(2.7 \%)$ had thalassemia, $6(1.3 \%)$ had renal cell cancer, and 3 had prostate cancer. Sickle cell disease, atrial fibrillation, breast cancer, gastric cancer and endometrial cancer were observed in two 
cases each. The association with thalassemia is noteworthy, as extramedullary hematopoiesis may occur in thalassemia, again hinting at a potential common pathogenic pathway in the two disease entities. Detailed patient characteristics, including bibliographical data for the case reports studied are included in Supplementary Table 1.

\section{Imaging features}

\section{Ultrasonography}

Myelolipoma is a benign tumor that contains adipose and hematopoietic tissue and while it expands it does not infiltrate the adjacent tissues. Accordingly a well-demarcated, hyperechoic and heterogenous mass can be seen growing out of the adrenal gland (Figure 3.). Calcification is often observed. The ultrasound picture of myelolipoma is diagnostically inadequate, and therefore further imaging by computed tomography or magnetic resonance imaging is necessary.

\section{Computed Tomography (CT)}

On CT scans, myelolipomas are well-circumscribed, round or elliptical, hypodense and heterogenous masses (Figure 4.). Attenuation values of -120 - -90 HU are characteristic of the adipose tissue, and the presence of fat density is useful and pivotal in diagnosing the mass as a myelolipoma (Figures $\mathbf{4 a}$ and $\mathbf{4 b}$ ). The average attenuation values vary because the proportion of the fatty component is variable. Atypical radiological features like hemorrhage, calcification can be seen [20]. On PET-CT, FDG uptake is usually absent [48], however the hematopoietic elements can rarely produce an intense ${ }^{18}$ fluorodeoxyglucose uptake [49]. 


\section{Magnetic Resonance Imaging}

On T1 and T2 weighted sequences, fat is hyperintense and well recognizable without fat suppression. The presence of fat can be confirmed with fat suppression. The tumors are almost always well-demarcated and heterogeneous. Boraschi et al. described three different morphologies on MRI when imaging myelolipomas (based on altogether six cases): 1) homogenous, hyperintense masses on $\mathrm{T} 1$ weighted sequences with intermediate signals on $\mathrm{T} 2$ weighted images; 2) heterogenous masses with fat intensity areas and hyperintense areas on T2 weighted images; 3 ) nodules hypointense to the liver on T1 weighted sequences and after gadolinium administration hyperintense on T2 weighted images suggesting tumors of myeloid elements [50]. A similar study with similar conclusions were presented again by Musante with 6 patients [51].

\section{Differential diagnosis}

It is very important to differentiate between myelolipomas and other lipomatous adrenal tumors. These masses include adrenocortical adenoma, adrenocortical carcinoma, retroperitoneal liposarcoma, exophytic renal angiomyolipoma and adrenal lipoma.

Adrenocortical adenomas are well-defined, homogenous, sharply marginated masses. To diagnose adrenocortical adenoma in routine clinical practice, an upper threshold value of $10 \mathrm{HU}$ was set for lipid rich adenomas that cover $70 \%$ of all ACAs. In $30 \%$ of the cases, the attenuation value is higher than $10 \mathrm{HU}$ pertaining to lipid-poor adenomas. Adenomas are isointense relative to liver and hyperintense relative to spleen on T1 weighted MRI [52]. 
Adrenocortical carcinomas (ACC) are usually large, in many cases lobulated tumors, which often have cystic, necrotic or hemorrhagic parts. ACCs are heterogeneous with irregular margins, can invade adjacent structures like liver, kidney, inferior vena cava. Attenuation values on $\mathrm{CT}$ are $>10 \mathrm{HU}$ in most cases. Due to tumor necrosis, low attenuation is visible in the central area. Peripheral nodular enhancement on contrast-enhanced CT is typical. The tumor gives low signal on T1 weighted MRI sequence and heterogeneously high signal on T2 weighted sequence. ${ }^{18}$ FDG-PET can be considered in the differential diagnosis $[53-55]$.

Renal angiomyolipoma is the most common benign mesenchymal tumor of the kidney, which is composed of mature fat, blood vessels and smooth muscle cells. It is a large necrotic tumor, and in the majority of the cases it extends through the renal capsule. On ultrasound, a homogenously echodense mass can be seen. CT image gives negative attenuation values. When a fat-containing solid mass growing out of kidney is seen, it is with high probability an angiomyolipoma. On T1 weighted MRI the tumor is hyperintense relative to the renal parenchyma and isointense relative to fat $[56,57]$

Retroperitoneal liposarcoma grows slowly, infiltrates the adjacent tissues and rarely gives metastasis. Three grades of aggressiveness are known histologically. The welldifferentiated lipogenic type is the most common form and is mostly similar to myelolipoma on radiological images. Contrast enhancement is visible on CT. Inhomogeneous and not welldemarcated mass is highly similar to myelolipoma [58].

The differential diagnosis of adrenal lipoma is only possible by the histological examination of the removed tumor, as it contains no myeloid elements. Adrenal lipoma is extremely rare (only 24 well-documented cases in the literature), and being also a benign 
tumor [39], its differential diagnosis from myelolipoma cannot be regarded as clinically relevant.

\section{Clinical management}

Due to the lack of formal guidelines regarding the management of adrenal myelolipomas, the decision should be made on a case-to-case basis. The majority of myelolipomas are found incidentally, not causing any trouble to the patient. These tumors are generally smaller than those producing symptoms due to mass effects. If the diagnosis of myelolipoma is unambiguous based on imaging, and there are no myelolipoma-related symptoms, no treatment is necessary. Surgical resection is the only treatment option if needed. Regarding adrenocortical adenomas, the European Society of Endocrinology's latest guideline proposes that if an incidentaloma is homogenous, its imaging features are consistent with a benign adrenal mass $(<10 \mathrm{HU})$ and smaller than $4 \mathrm{~cm}$ in diameter, no further imaging is needed [55, 59, 60]. Myelolipomas, on the other hand, are usually larger tumors, but also slowly growing. Due to the lack of sufficient data, it would be difficult to determine the threshold diameter, above which imaging by follow-up could be proposed. Follow-up of giant myelolipomas (diameter $>10 \mathrm{~cm}$ ), however, may be warranted, but the frequency for follow-up is yet to be determined. Being larger than adrenocortical adenomas, follow-up of AMLs by ultrasonography might be feasible.

The issue on the necessity of hormonal evaluation of AML is debated. In the AACE guideline, Zeiger et al. did not propose hormonal evaluation for obvious myelolipoma [61]. Similarly, Terzolo et al. propose no routine hormonal work-up for patients with AML, either [62]. In contrast, Shenoy et al. argued for metabolic work-up in patients with AML [20]. According to our data, $18.3 \%$ of all adrenal myelolipomas are associated with some kind of 
endocrine disorder, including congenital adrenal hyperplasia (in $10 \%$ ), and others like primary aldosteronism, hypercortisolism or hyperandrogenism. The clinical picture should be considered to be the major factor in determining the necessity for hormonal work-up.

If surgical removal is indicated because of significant growth, symptoms (e.g. abdominal discomfort) or hormonal activity, laparotomy or laparoscopy can be performed. Laparoscopic adrenalectomy is safe and effective [63]. Two approaches are applied at laparoscopic surgery, the transperitoneal and retroperitoneal approach. Laparoscopic myelolipoma removal is possible even for cases of giant myelolipoma. Chaudhary et al. reported a case, wherein a $15 \times 11 \mathrm{~cm}$ myelolipoma was removed successfully through a 2.5 $\mathrm{cm}$ incision [64]. In another report, two giant myelolipomas of 11 and $14 \mathrm{~cm}$ were removed without complication by laparoscopic approach [65]. Large tumors can be removed much more easily by the transperitoneal approach than by retroperitoneal approach [66]. Shen et al. described a retroperitoneal approach for operating large myelolipomas by a technique including liposuction [67]. Two reports demonstrated that if a myelolipoma ruptures, transcatheter arterial embolization using gelatin sponge particles can solve retroperitoneal bleeding from the tumor, and can delay the definitive tumor removal to an elected operation $[68,69]$.

\section{Conclusions}

Adrenal myelolipoma is an invariably benign tumor composed of adipose and extramedullary hematopoietic tissue elements. It is usually a large tumor most often diagnosed in the sixth decade of life. Due to the variable proportion of fat and hematopoietic elements, adrenal myelolipoma might cause sometimes severe differential diagnostic problems mostly with adrenocortical cancer. Detailed imaging including CT and MRI might be helpful. If its 
diagnosis is unambiguous, and no symptoms are associated, adrenal myelolipoma mostly requires no surgical intervention.

\section{Declaration of interest}

The authors state that there are no conflicts of interest.

\section{Acknowledgements}

The study has been supported by a grant from the Hungarian National Research, Development and Innovation Office (NKFIH K115398) to Dr. Peter Igaz. The authors would like to thank Dr. Katalin Borka MD PhD for providing a microscopic picture of myelolipoma and pathologic data of our case series. 


\section{References:}

1. Lam, A.K.: Update on Adrenal Tumours in 2017 World Health Organization (WHO) of Endocrine Tumours. Endocr. Pathol. 1-15 (2017). doi:10.1007/s12022-017-9484-5

2. Olsson, C.A., Krane, R.J., Klugo, R.C., Selikowitz, S.M.: Adrenal myelolipoma. Surgery. 73, 665-70 (1973)

3. Song, J.H., Chaudhry, F.S., Mayo-Smith, W.W.: The Incidental Adrenal Mass on CT: Prevalence of Adrenal Disease in 1,049 Consecutive Adrenal Masses in Patients with No Known Malignancy. Am. J. Roentgenol. 190, 1163-1168 (2008). doi:10.2214/AJR.07.2799

4. Bin, X., Qing, Y., Linhui, W., Li, G., Yinghao, S.: Adrenal incidentalomas: Experience from a retrospective study in a Chinese population. URO. 29, 270-274 (2011). doi:10.1016/j.urolonc.2009.03.027

5. Mantero, F., Masini, A.M., Opocher, G., Giovagnetti, M., Arnaldi, G., Tumors, on behalf of the N.I.S.G. on A.: Adrenal Incidentaloma: An Overview of Hormonal Data from the National Italian Study Group. Horm. Res. 47, 284-289 (1997). doi:10.1159/000185478

6. Minamiya, Y., Abo, S., Kitamura, M., Izumi, K.: Mediastinal extraadrenal myelolipoma: report of a case. Surg. Today. 27, 971-2 (1997)

7. Zeng, Y., Ma, Q., Lin, L., Fu, P., Shen, Y., Luo, Q.-Y., Zhao, L.-H., Mou, J.-H., Xiao, H.-L.: Giant Myelolipoma in the Spleen: A Rare Case Report and Literature Review. Int. J. Surg. Pathol. 24, 177-180 (2016). doi:10.1177/1066896915617027

8. Ghaouti, M., Znati, K., Jahid, A., Zouaidia, F., Bernoussi, Z., Mahassini, N.: Renal myelolipoma: a rare extra-adrenal tumor in a rare site: a case report and review of the literature. J. Med. Case Rep. 7, 92 (2013). doi:10.1186/1752-1947-7-92

9. Sundaram, M., Bauer, T., von Hochstetter, A., Ilaslan, H., Joyce, M.: Intraosseous myelolipoma. Skeletal Radiol. 36, 1181-1184 (2007). doi:10.1007/s00256-007-0347-x

10. Krismann, M., Reichle, G., Müller, K.M.: [Thoracic bilateral myelolipoma]. Pneumologie. 47, 501-3 (1993)

11. George, S.A., Manipadam, M.T., Thomas, R.: Primary myelolipoma presenting as a nasal cavity polyp: a case report and review of the literature. J. Med. Case Rep. 6, 127 (2012). doi:10.1186/1752-1947-6-127

12. Stein, S.H., Latour, F., Frost, S.S.: Myelolipoma arising from ectopic adrenal cortex: case report and review of the literature. Am. J. Gastroenterol. 81, 999-1001 (1986)

13. Cina, S.J., Gordon, B.M., Curry, N.S.: Ectopic adrenal myelolipoma presenting as a splenic mass. Arch. Pathol. Lab. Med. 119, 561-3 (1995)

14. Newman, S.J., Inzana, K., Chickering, W.: Extradural Myelolipoma in a Dog. J. Vet. Diagnostic Investig. 12, 71-74 (2000). doi:10.1177/104063870001200115

15. Storms, G., Janssens, G.: Intraocular myelolipoma in a dog. Vet. Ophthalmol. 16, $183-$ 187 (2013). doi:10.1111/vop.12059

16. Taher, A., Vichinsky, E., Musallam, K., Cappellini, M.D., Viprakasit, V., Weatherall, S.D.: Guidelines for the Management of Non Transfusion Dependent Thalassaemia 
(NTDT). Thalassaemia International Federation (2013)

17. Fowler, M.R., Williams, R.B., Alba, J.M., Byrd, C.R.: Extra-adrenal myelolipomas compared with extramedullary hematopoietic tumors: a case of presacral myelolipoma. Am. J. Surg. Pathol. 6, 363-74 (1982)

18. Chen, K.T.K., Felix, E.L., Flam, M.S.: Extraadrenal Myelolipoma. Am. J. Clin. Pathol. $78,(1982)$

19. Sanders, R., Bissada, N., Curry, N., Gordon, B.: Clinical Spectrum of Adrenal Myelolipoma: Analysis of 8 Tumors in 7 Patients. J. Urol. 153, 1791-1793 (1995). doi:10.1016/S0022-5347(01)67307-5

20. Shenoy, V.G., Thota, A., Shankar, R., Desai, M.G.: Adrenal myelolipoma: Controversies in its management. Indian J. Urol. 31, 94-101 (2015). doi:10.4103/09701591.152807

21. De Navasquez, S.: Case of myelo-lipoma (bone-marrow, heterotopia) pf suprarenal gland. Guys. Hosp. Rep. 88, 237-240 (1935)

22. Chang, K.-C., Chen, P.-I., Huang, Z.-H., Lin, Y.-M., Kuo, P.-L.: Adrenal myelolipoma with translocation (3;21)(q25;p11). Cancer Genet. Cytogenet. 134, 77-80 (2002). doi:10.1016/S0165-4608(01)00592-1

23. Bishop, E., Eble, J.N., Cheng, L., Wang, M., Chase, D.R., Orazi, A., O??Malley, D.P.: Adrenal Myelolipomas Show Nonrandom X-chromosome Inactivation in Hematopoietic Elements and Fat: Support for a Clonal Origin of Myelolipomas. Am. J. Surg. Pathol. 30, 838-843 (2006). doi:10.1097/01.pas.0000202044.05333.17

24. Feng, C., Jiang, H., Ding, Q., Wen, H.: Adrenal myelolipoma: A mingle of progenitor cells? Med. Hypotheses. 80, 819-822 (2013). doi:10.1016/j.mehy.2013.03.021

25. Selye, H., Stone, H.: Hormonally induced transformation of adrenal into myeloid tissue. Am. J. Pathol. 26, 211-33 (1950)

26. Sieber, S.C., Gelfman, N.A., Dandurand, R., Braza, F.: Ectopic ACTH and adrenal myelolipoma. Conn. Med. 53, 7-10 (1989)

27. Hagiwara, H., Usui, T., Kimura, T., Tagami, T., Naruse, M., Minamiguchi, S., Kato, T., Okuno, H., Shimatsu, A.: Lack of ACTH and Androgen Receptor Expression in a Giant Adrenal Myelolipoma Associated with 21-hydroxylase Deficiency. Endocr. Pathol. 19, 122-127 (2008). doi:10.1007/s12022-008-9034-2

28. Motta, I., Boiocchi, L., Delbini, P., Migone De Amicis, M., Cassinerio, E., Dondossola, D., Rossi, G., Cappellini, M.D.: A giant adrenal myelolipoma in a beta-thalassemia major patient: Does ineffective erythropoiesis play a role? Am. J. Hematol. 91, 12811282 (2016). doi:10.1002/ajh.24446

29. Rubin, B.P., Allison, K.H., Mann, G.N., Norwood, T.H.: An Unusual Case of Multiple Giant Myelolipomas: Clinical and Pathogenetic Implications. Endocr. Pathol. 14, 93100 (2003)

30. Merchant, S.H., Herman, C.M., Amin, M.B., Ro, J.Y., Troncoso, P.: Myelolipoma Associated With Adrenal Ganglioneuroma. Arch Pathol Lab Med-Vol. 126, (2002)

31. Lu, H., Gan, M., Chen, H., Huang, S.: Adrenal myelolipoma within myxoid cortical adenoma associated with Conn's syndrome. J. Zhejiang Univ. Sci. B. 9, 500-5 (2008). doi:10.1631/jzus.B0720014 
32. Kumar, U., Priyadarshi, S., Tomar, V., Vohra, R.R.: Adrenal myelolipoma with osseous metaplasia and hypercortisolism. Urol. Ann. 9, 71-73 (2017). doi:10.4103/0974-7796.198841

33. Armand, R., Cappola, A.R., Horenstein, R.B., Drachenberg, C.B., Sasano, H., Papadimitriou, J.C.: Adrenal Cortical Adenoma with Excess Black Pigment Deposition, Combined with Myelolipoma and Clinical Cushing's Syndrome: A Case Report and Review of the Literature. Int. J. Surg. Pathol. 12, 57-61 (2004). doi:10.1177/106689690401200111

34. Schwartz, B.F., Wasson, L.: Hibernoma arising from the adrenal gland. Urology. 61, 1035 (2003). doi:10.1016/S0090-4295(02)02586-4

35. Cardinalli, I.A., de Oliveira-Filho, A.G., Mastellaro, M.J., Ribeiro, R.C., Aguiar, S.S.: A unique case of synchronous functional adrenocortical adenoma and myelolipoma within the ectopic adrenal cortex in a child with Beckwith-Wiedemann syndrome. Pathol. - Res. Pract. 208, 189-194 (2012). doi:10.1016/j.prp.2011.12.011

36. Sebastià, M.C., Pérez-Molina, M.O., Alvarez-Castells, A., Quiroga, S., Pallisa, E.: CT evaluation of underlying cause in spontaneous subcapsular and perirenal hemorrhage. Eur. Radiol. 7, 686-690 (1997). doi:10.1007/BF02742926

37. Low, G., Dhliwayo, H., Lomas, D.J.: Adrenal neoplasms. Clin. Radiol. 67, 988-1000 (2012). doi:10.1016/j.crad.2012.02.005

38. Lam, K.Y., Lo, C.Y.: Adrenal lipomatous tumours: a 30 year clinico- pathological experience at a single institution. J Clin Pathol. 54, 707-712 (2001)

39. Lam, A.K.: Lipomatous tumours in adrenal gland: WHO updates and clinical implications. Endocr. Relat. Cancer. 24, R65-R79 (2017). doi:10.1530/ERC-16-0564

40. Han, M., Burnett, A.L., Fishman, E.K., Marshall, F.F.: The Natural History and Treatment of Adrenal Myelolipoma. J. Urol. 157, 1213-1216 (1997). doi:10.1016/S0022-5347(01)64926-7

41. Campbell, M.J., Obasi, M., Wu, B., Corwin, M.T., Fananapazir, G.: The radiographically diagnosed adrenal myelolipoma: what do we really know? Endocrine. 1-6 (2017). doi:10.1007/s12020-017-1410-6

42. Liu, H.-P., Chang, W.-Y., Chien, S.-T., Hsu, C.-W., Wu, Y.-C., Kung, W.-C., Su, C.M., Liu, P.-H.: Intra-abdominal bleeding with hemorrhagic shock: a case of adrenal myelolipoma and review of literature. BMC Surg. 17, 74 (2017). doi:10.1186/s12893017-0270-6

43. Reza-Albarran, A.A., Gomez-Perez, F.J., Lopez, J.C., Herrera, M., GamboaDominguez, A., Keirns, C., Aranda, A., Rull, J.A.: Myelolipoma: A New Adrenal Finding in Carney's Complex? Endocr. Pathol. 10, 251-257 (1999)

44. Kalafatis, P.: Bilateral Giant Adrenal Myelolipoma and Polycystic Ovarian Disease. Urol Int. 63, 139-143 (1999)

45. Yoshioka, M., Fujimori, K., Wakasugi, M., Yamazaki, N., Kuroki, M., Tsuchida, T., Seki, H., Sekiya, M., Tamura, T.: Cushing's disease associated with adrenal myelolipoma, adrenal calcification and thyroid cancer. Endocr. J. 41, 461-6 (1994)

46. Banik, S., Hasleton, P.S., Lyon, R.L.: An unusual variant of multiple endocrine neoplasia syndrome: a case report. Histopathology. 8, 135-44 (1984) 
47. Maschler, I., Rosenmann, E., Ehrenfeld, E.N.: Ectopic functioning adrenocorticomyelolipoma in longstanding Nelson's syndrome. Clin. Endocrinol. (Oxf). 10, 493-7 (1979)

48. Gemmel, F., Bruinsma, H., Oomen, P., Collins, J.: PET/CT Incidental Detection of Bilateral Adrenal Myelolipomas in a Patient With a Huge Maxillary Sinus Carcinoma. Clin. Nucl. Med. 35, 132-133 (2010). doi:10.1097/RLU.0b013e3181c7c007

49. Ludwig, V., Rice, M.H., Martin, W.H., Kelley, M.C., Delbeke, D.: 2-Deoxy-2[18F]fluoro-D-glucose positron emission tomography uptake in a giant adrenal myelolipoma. Mol. Imaging Biol. 4, 355-8 (2002)

50. Boraschi, P., Braccini, G., Gigoni, R., Cartei, F., Campatelli, A., Di Vito, A., Perri, G.: [Adrenal myelolipomas: their magnetic resonance assessment]. Clin. Ter. 147, 549-57 (1996)

51. Musante, F., Derchi, L.E., Bazzocchi, M., Avataneo, T., Gandini, G., Pozzi Mucelli, R.S.: MR imaging of adrenal myelolipomas. J. Comput. Assist. Tomogr. 15, 111-4

52. Wale, D.J., Wong, K.K., Viglianti, B.L., Rubello, D., Gross, M.D.: Contemporary imaging of incidentally discovered adrenal masses. Biomed. Pharmacother. 87, 256262 (2017). doi:10.1016/j.biopha.2016.12.090

53. Fassnacht, M., Kroiss, M., Allolio, B.: Update in Adrenocortical Carcinoma. J. Clin. Endocrinol. Metab. 98, 4551-4564 (2013). doi:10.1210/jc.2013-3020

54. Nunes, M.L., Rault, A., Teynie, J., Valli, N., Guyot, M., Gaye, D., Belleannee, G., Tabarin, A.: 18F-FDG PET for the Identification of Adrenocortical Carcinomas among Indeterminate Adrenal Tumors at Computed Tomography Scanning. World J. Surg. 34, 1506-1510 (2010). doi:10.1007/s00268-010-0576-3

55. Fassnacht, M., Arlt, W., Bancos, I., Dralle, H., Newell-Price, J., Sahdev, A., Tabarin, A., Terzolo, M., Tsagarakis, S., Dekkers, O.M.: Management of adrenal incidentalomas: European Society of Endocrinology Clinical Practice Guideline in collaboration with the European Network for the Study of Adrenal Tumors. Eur. J. Endocrinol. 175, G1-G34 (2016). doi:10.1530/EJE-16-0467

56. Zhong, Y., Shen, Y., Pan, J., Wang, Y., An, Y., Guo, A., Ma, L., Ye, H., Wang, H.: Renal epithelioid angiomyolipoma: MRI findings. Radiol. Med. (2017). doi:10.1007/s11547-017-0788-9

57. Krishna, S., Murray, C.A., McInnes, M.D., Chatelain, R., Siddaiah, M., Al-Dandan, O., Narayanasamy, S., Schieda, N.: CT imaging of solid renal masses: pitfalls and solutions. Clin. Radiol. 72, 708-721 (2017). doi:10.1016/j.crad.2017.05.003

58. Craig, W.D., Fanburg-Smith, J.C., Henry, L.R., Guerrero, R., Barton, J.H.: Fatcontaining Lesions of the Retroperitoneum: Radiologic-Pathologic Correlation. RadioGraphics. 29, 261-290 (2009). doi:10.1148/rg.291085203

59. Terzolo, M., Bovio, S., Pia, A., Reimondo, G., Angeli, A.: Management of adrenal incidentaloma. Best Pract. Res. Clin. Endocrinol. Metab. 23, 233-243 (2009). doi:10.1016/j.beem.2009.04.001

60. Libè, R., Dall'Asta, C., Barbetta, L., Baccarelli, A., Beck-Peccoz, P., Ambrosi, B.: Long-term follow-up study of patients with adrenal incidentalomas. Eur. J. Endocrinol. 147, 489-94 (2002) 
61. Zeiger, M., Thompson, G., Duh, Q.-Y., Hamrahian, A., Angelos, P., Elaraj, D., Fishman, E., Kharlip, J., American Association of Clinical Endocrinologists, American Association of Endocrine Surgeons: American Association of Clinical Endocrinologists and American Association of Endocrine Surgeons Medical Guidelines for the Management of Adrenal Incidentalomas: Executive Summary of Recommendations. Endocr. Pract. 15, 450-453 (2009). doi:10.4158/EP.15.5.450

62. Terzolo, M., Stigliano, A., Chiodini, I., Loli, P., Furlani, L., Arnaldi, G., Reimondo, G., Pia, A., Toscano, V., Zini, M., Borretta, G., Papini, E., Garofalo, P., Allolio, B., Dupas, B., Mantero, F., Tabarin, A.: AME Position Statement on adrenal incidentaloma. Eur. J. Endocrinol. 164, 851-870 (2011). doi:10.1530/EJE-10-1147

63. Yamashita, S., Ito, K., Furushima, K., Fukushima, J., Kameyama, S., Harihara, Y.: Laparoscopic versus open adrenalectomy for adrenal myelolipoma. Ann. Med. Surg. 3, 34-8 (2014). doi:10.1016/j.amsu.2014.04.001

64. Chaudhary, R., Deshmukh, A., Singh, K., Biswas, R.: Is size really a contraindication for laparoscopic resection of giant adrenal myelolipomas? BMJ Case Rep. 2016, bcr2016215048 (2016). doi:10.1136/bcr-2016-215048

65. Agrusa, A., Romano, G., Frazzetta, G., Chianetta, D., Sorce, V., Di Buono, G., Gulotta, G.: Laparoscopic adrenalectomy for large adrenal masses: Single team experience. Int. J. Surg. 12, S72-S74 (2014). doi:10.1016/j.ijsu.2014.05.050

66. Hemal, A.K., Singh, A., Gupta, N.P.: Whether adrenal mass more than $5 \mathrm{~cm}$ can pose problem in laparoscopic adrenalectomy? An evaluation of 22 patients. World J Urol. 26, 505-508 (2008). doi:10.1007/s00345-008-0270-3

67. Shen, X., Qiu, Y., Zheng, Y., Zhang, S.: Retroperitoneal Laparoscopic Liposuction for Large Adrenal Myelolipomas: A Report of Nine Cases. J. Laparoendosc. Adv. Surg. Tech. 22, 578-580 (2012). doi:10.1089/lap.2012.0113

68. Nakajo, M., Onohara, S., Shinmura, K., Fujiyoshi, F., Nakajo, M.: Embolization for spontaneous retroperitoneal hemorrhage from adrenal myelolipoma. Radiat. Med. 21, 214-9

69. Chng, S.M., Lin, M.B.K., Ng, F.C., Chng, H.C., Khoo, T.K.: Adrenal myelolipoma presenting with spontaneous retroperitoneal haemorrhage demonstrated on computed tomography and angiogram--a case report. Ann. Acad. Med. Singapore. 31, 228-30 (2002) 


\section{Legends for figures:}

Figure 1. a. Correlation between tumor diameter and tumor weight, Pearson correlation test was used; b. microscopic image of an adrenal myelolipoma. 200x magnification. Courtesy of Dr. Katalin Borka MD PhD, $2^{\text {nd }}$ Department of Pathology, Semmelweis University

Figure 2. The age of patients at the diagnosis of AML.

Figure 3. A representative ultrasonographic image of a left-sided giant myelolipoma

Figure 4. a. CT image of a right-sided giant adrenal myelolipoma; b. CT image of a bilateral adrenal myelolipoma with calcifications 
Table 1. Reported leading complaints of myelolipoma patients.

\begin{tabular}{|c|c|c|}
\hline Leading complaint & $\begin{array}{c}\text { Number of patients } \\
\text { with complaint }\end{array}$ & Percentage $(\%)$ \\
\hline Abdominal discomfort/pain & 60 & 22.5 \\
\hline Hypochondrial pain & 37 & 13.9 \\
\hline Flank pain & 37 & 13.9 \\
\hline Abdominal mass & 14 & 5.2 \\
\hline Dyspepsia & 13 & 4.9 \\
\hline Nausea/vomiting & 12 & 4.5 \\
\hline Loin pain & 11 & 4.1 \\
\hline Sudden abdominal/flank pain & 9 & 3.4 \\
\hline Intermittent pain & 8 & 3.0 \\
\hline $\begin{array}{l}\text { Lower extremity paresthesia, } \\
\text { edema, paralysis }\end{array}$ & 8 & 3.0 \\
\hline Epigastric pain & 6 & 2.2 \\
\hline Shortness of breath & 5 & 1.9 \\
\hline Back pain & 4 & 1.5 \\
\hline Weight loss & 4 & 1.5 \\
\hline Fever & 4 & 1.5 \\
\hline Chest pain & 3 & 1.1 \\
\hline Virilisation & 1 & 0.4 \\
\hline Dragging sensation & 1 & 0.4 \\
\hline Generalized edema & 1 & 0.4 \\
\hline Other (below 3\%) & 29 & 10.9 \\
\hline Total & 267 & 100.0 \\
\hline
\end{tabular}


Fig. 1.

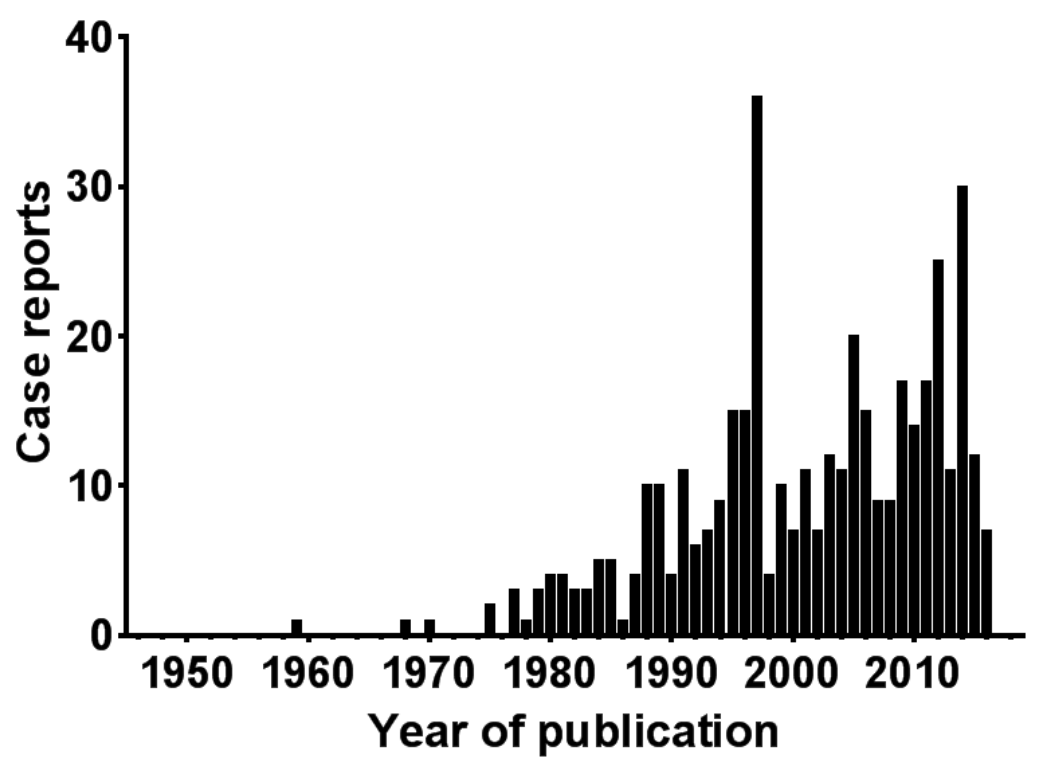


Fig. 2.

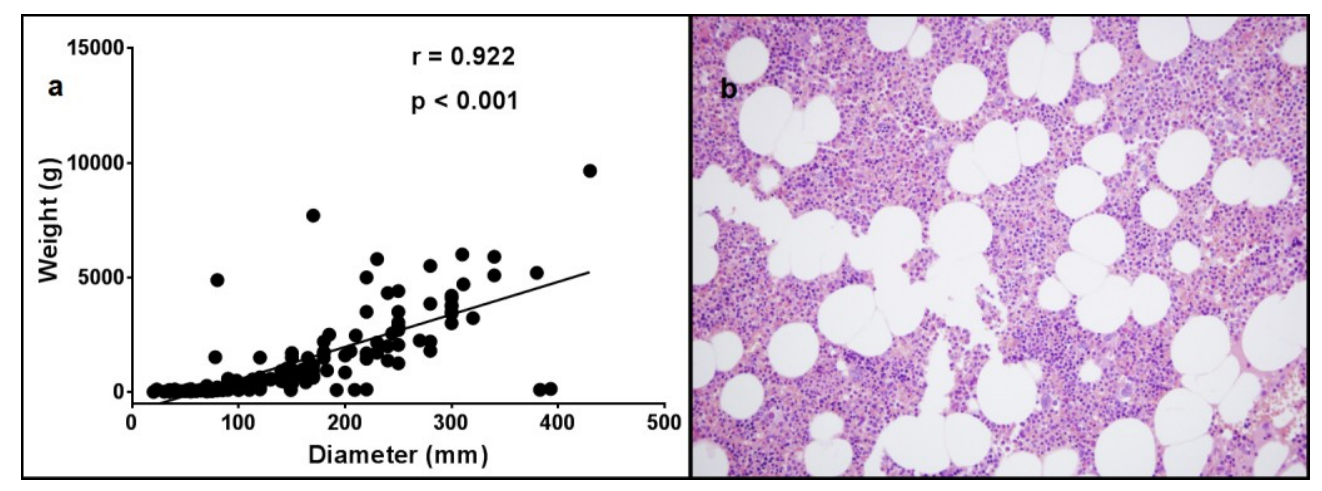


Fig. 3.

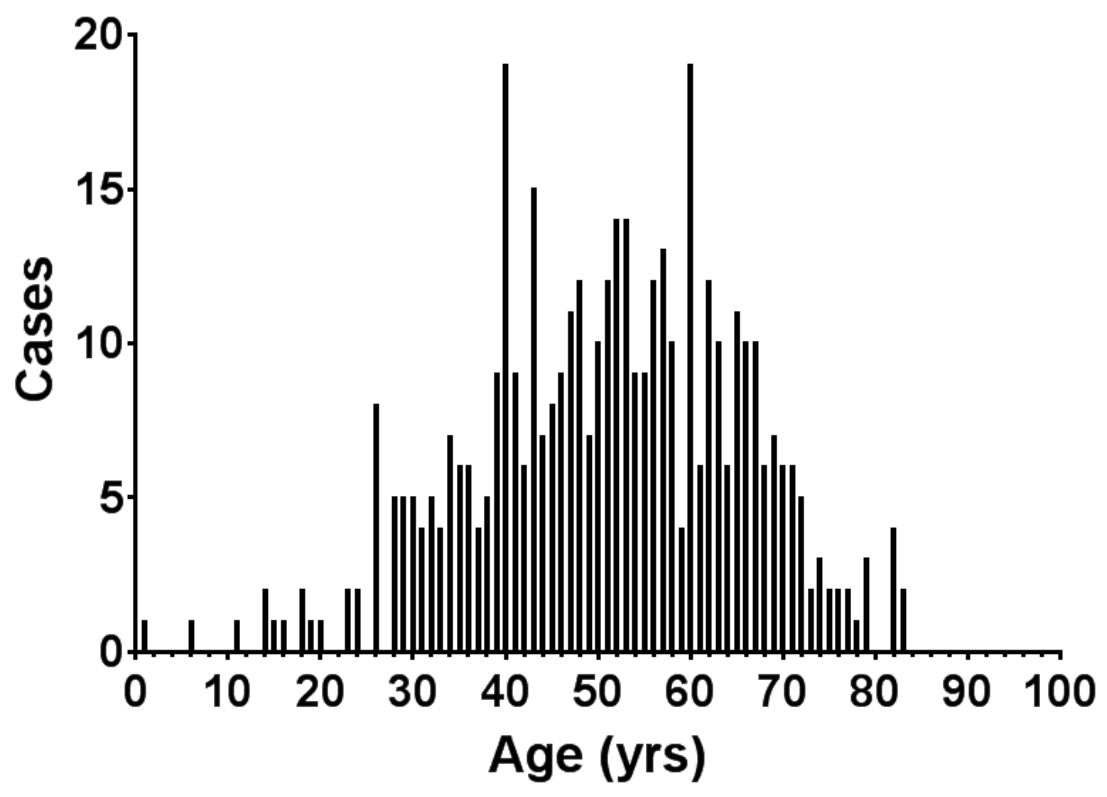


Fig. 4.

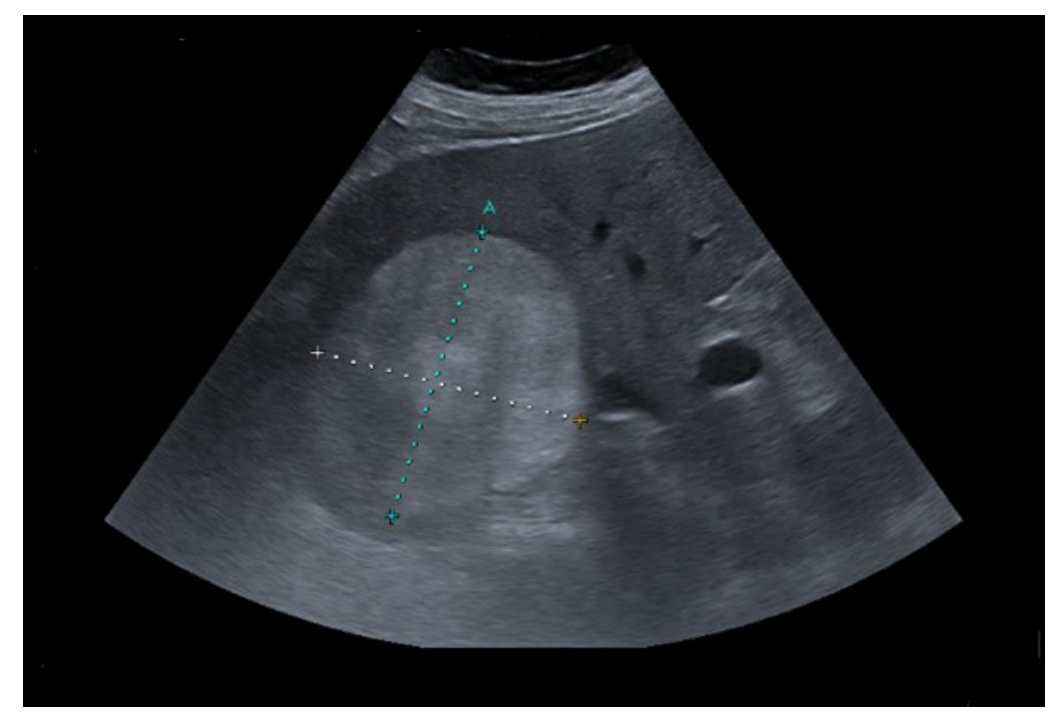


Fig. 5.

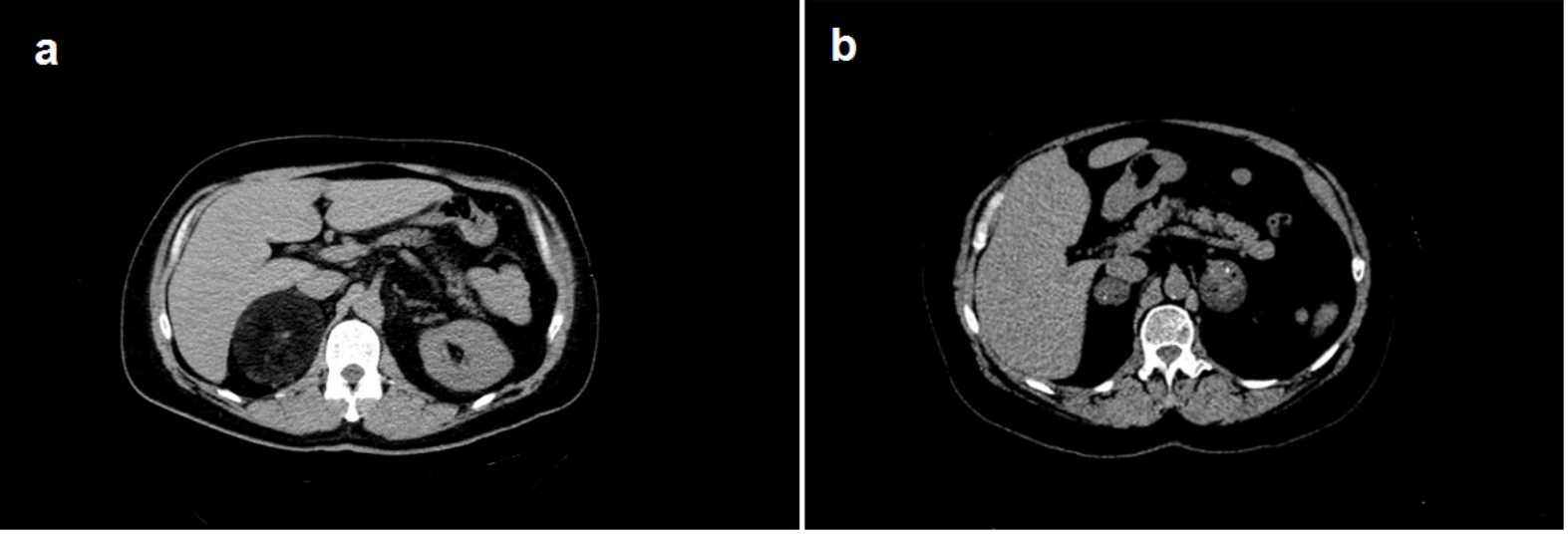

